\title{
Photoluminescence of molecular $\mathrm{C}_{70}$ at $1.5 \mathrm{~K}$. On the nature of the lowest excited states
}

\author{
J.B.M. Warntjes ${ }^{\text {a }}$, I. Holleman ${ }^{\text {b }}$, G. Meijer ${ }^{\text {b }}$, E.J.J. Groenen ${ }^{\text {a }}$ \\ a Centre for the study of Excited States of Molecules, Huygens Laboratory, University of Leiden, P.O. Box 9504, 2300 RA Leiden, \\ The Netherlands \\ ' Department of Molecular and Laser Physics, University of Nijmegen, Toernooiveld, 6525 ED Nijmegen, The Netherlands
}

Received 1 July 1996

\begin{abstract}
We report Shpol'skii-type luminescence spectra of $C_{70}$ in n-pentane at $1.5 \mathrm{~K}$ with vibronic linewidths of $3 \mathrm{~cm}^{-1}$. The vibronic structure of both fluorescence and phosphorescence has been unravelled. The fluorescence intensity largely shows up in vibronic bands corresponding to transitions to ground-state infrared-active vibrational modes and it is concluded that the first excited singlet state of $C_{70}$ in alkanes is of $A_{2}^{\prime}$ character.
\end{abstract}

\section{Introduction}

The investigation of the lowest electronically excited states of fullerenes like $C_{60}$ and $C_{70}$ is both interesting and difficult because of the large density of close-lying and mostly degenerate states. For $\mathrm{C}_{70}$ the electronic structure of the lowest excited states of singlet and triplet multiplicity is an open question. In quantum-chemical calculations on $\mathrm{C}_{70}$ of $\mathrm{D}_{5 \mathrm{~h}}$ symmetry by Hückel and Hartree-Fock methods the LUMO (lowest unoccupied molecular orbital) and LUMO +1 orbitals were found too close in energy to infer which one is lowest $[1,2]$. The orbital character of the lowest excited state is predicted to be either $A_{2}^{\prime}$ or $E_{1}^{\prime}$ corresponding to, respectively, an electric-dipole forbidden and a (weakly) allowed transition to the ground state. From INDO/CI calculations Feng et al. [3] concluded to an $S_{1}$ of $A_{2}^{\prime}$ symmetry. Fulara et al. [4] suggested that the vibra- tional structure of the $S_{1} \leftarrow S_{0}$ absorption of $C_{70}$ in a neon matrix is indicative of an $S_{1}$ of $E_{1}^{\prime}$ symmetry, while Leach [5] proposed an alternative assignment of this structure corresponding to an $\mathrm{S}_{1}$ of $\mathrm{A}_{2}^{\prime}$ symmetry.

The long-wavelength absorption derives from a number of overlapping transitions which suggests that the study of the luminescence may well provide an easier access to the nature of the lowest excited states. Indeed, for the icosahedral $\mathrm{C}_{60}$ molecule we have recently reported well-resolved fluorescence spectra both from solution and from single crystals at $1.2 \mathrm{~K}$ [6]. Analysis of the vibronic intensity distribution led us to conclude that the first excited singlet state of molecular $\mathrm{C}_{60}$ has a mixed $\mathrm{T}_{1 \mathrm{~g}}, \mathrm{G}_{\mathrm{g}}$, and $\mathrm{T}_{2 \mathrm{~g}}$ character. Among a number of low-temperature luminescence studies on $\mathrm{C}_{70}$ [7-14], those in a frozen methylcyclohexane solution [10-13] and in a crystalline toluene matrix [14] have led to well-resolved 
fluorescence and phosphorescence spectra. The fluorescence in methylcyclohexane extends from about 15600 down to $12650 \mathrm{~cm}^{-1}$ where it gets overshadowed by the more intense phosphorescence whose vibronic structure could be followed down to about $10600 \mathrm{~cm}^{-1}$. The positions of both the $S_{1} \rightarrow S_{0}$ and $\mathrm{T}_{1} \rightarrow \mathrm{S}_{0}$ electronic origins were determined and the intensity of these $0-0$ transitions relative to that of the vibronic bands was reported to depend strongly on the solvent [13]. In this study the vibronic structure was largely assigned to $e_{2}^{\prime}$ modes and $S_{1}$ and $T_{1}$ were identified as of $E_{1}^{\prime}$ symmetry.

Both theoretical and experimental limitations as yet preclude definite conclusions regarding the nature of the lower excited states. On the one hand, calculations of the expected vibronic intensity patterns of the $S_{1} \rightarrow S_{0}$ and $T_{1} \rightarrow S_{0}$ transitions including the activity of Herzberg-Teller and Jahn-Teller active modes are missing. On the other hand, a measurement of the fully resolved vibronic structure of the luminescence is necessary. In this Letter we describe luminescence spectra in three n-alkanes at liquid-helium temperatures and show that under fast cooling of a solution of $\mathrm{C}_{70}$ in n-pentane luminescence spectra are obtained with vibronic lines of about $3 \mathrm{~cm}^{-1}$ width. Besides the $S_{1} \rightarrow S_{0}$ and $T_{1} \rightarrow$ $\mathrm{S}_{0}$ origins, these Shpol'skii-type spectra reveal 12 vibronic bands in the fluorescence and 19 in the phosphorescence with a relative intensity larger than $2 \%$ of the most intense band. We show that the occurrence of many vibronic bands in the fluorescence corresponding to transitions to ground-state vibrational levels involving infrared-active modes refers to the activity of Herzberg-Teller active modes in inducing fluorescence. Analysis of this vibronic coupling leads us to conclude that $S_{1}$ of $C_{70}$ in alkanes is of $A_{2}^{\prime}$ character.

\section{Experimental}

Highly purified $\mathrm{C}_{70}(\geqslant 99.9 \%)$ was dissolved in sodium-dried $n$-heptane, $n$-hexane and $n$-pentane. The solutions were degassed and sealed in suprasil tubes with a diameter of $4 \mathrm{~mm}$. Two methods of cooling were applied. Quick cooling consisted of plunging the sample directly into liquid helium. Slow cooling was achieved by immersing the sample tubes into liquid nitrogen under a controlled rate of the order of centimeters per hour. The samples were then transferred into a liquid-helium filled cryostat.

For excitation either the $514 \mathrm{~nm}$ line of an $\mathrm{Ar}^{+}$ laser, from which all other lines were suppressed using a band filter, or an $\mathrm{Ar}^{+}$pumped dye laser was used. Laser fluence was always kept low, with a maximum of $3 \mathrm{~mW} / \mathrm{mm}^{2}$. The emission of the sample was focussed onto the entrance slit of a $1 \mathrm{~m}$ monochromator and detected using an optical multichannel analyser (EG\&G OMA-vision-CCD). Each $\mathrm{CCD}$ read-out in the region of interest covered about $200 \mathrm{~cm}^{-1}$ with an effective resolution of about 0.5 $\mathrm{cm}^{-1}$. We calibrated all of these read-outs using a neon calibration lamp and merged them with specially designed software, which included a correction for the wavelength dependence of the quantum efficiency of the CCD.

\section{Results and discussion}

In Fig. 1 luminescence spectra at $1.5 \mathrm{~K}$, upon excitation at $514 \mathrm{~nm}$, are shown of frozen solutions of $5 \times 10^{-6} \mathrm{M} \mathrm{C}_{70}$ in $\mathrm{n}$-heptane, $\mathrm{n}$-hexane, and n-pentane. The top spectrum concerns n-heptane (Fig. 1a). The fluorescence origin occurs at 15242 $\mathrm{cm}^{-1}$, the phosphorescence origin at $12455 \mathrm{~cm}^{-1}$ and all bands have full-width at half-maximum somewhat less than $100 \mathrm{~cm}^{-1}$. The spectrum is comparable to well-resolved spectra in similar solvents reported in the literature, e.g., those in methylcyclohexane [10-13]. For n-hexane, Fig. 1b, the spectrum looks quite different. While some relatively narrow features may still be recognized, these are superimposed upon a broad spectrum with a first band at $14740 \mathrm{~cm}^{-1}$. In contrast to the spectrum from the n-heptane solution, that from the n-hexane solution depends on the rate of cooling and the position in the sample tube. A slow cooling results in a large contribution of the broad spectrum for irradiation of the centre of the tube, as in Fig. 1b. A similarly unstructured spectrum has been observed upon slow cooling of a solution in n-pentane, the first band being slightly narrower and shifted to $14830 \mathrm{~cm}^{-1}$. As shown in Fig. 1c, for n-pentane sufficiently slow cooling may even lead to the virtual absence of the narrow-band spectrum. We attribute 

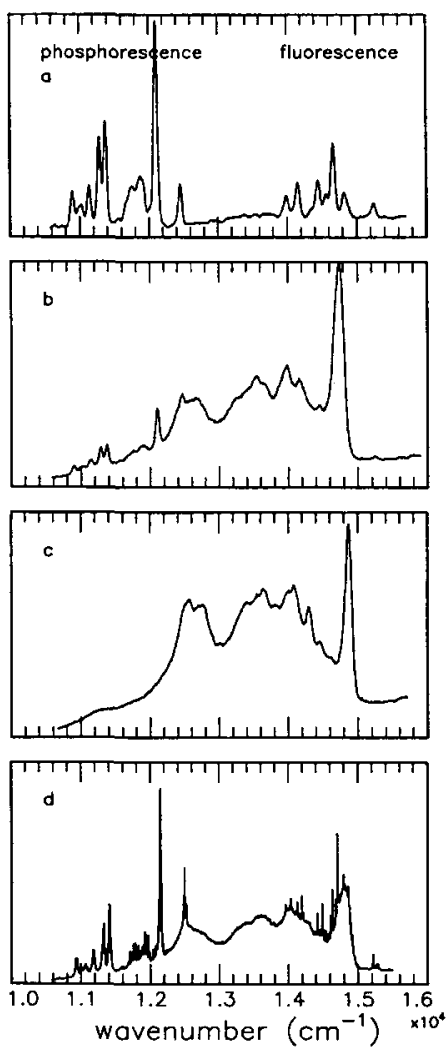

Fig. 1. The emission at $1.5 \mathrm{~K}$ of a $5 \times 10^{-6} \mathrm{M}$ solution of $\mathrm{C}_{70}$ in (a) n-heptane (slowly cooled), (b) n-hexane (slowly cooled), (c) n-pentane (slowly cooled), and (d) n-pentane (quickly cooled). Excitation at $514 \mathrm{~nm}$.

the broad luminescence spectra (e.g., that in Fig. 1c) to clusters of $\mathrm{C}_{70}$ molecules because of their occurrence upon slow cooling and the low solubility of $\mathrm{C}_{70}$ in n-alkanes.

A more interesting observation follows upon fast cooling of the n-pentane solution (Fig. 1d). The broad spectrum is still there and in addition a spectrum consisting of narrow bands shows up both in the fluorescence and in the phosphorescence region. A closer look reveals that these bands consist of repetitive multiplet structures. Such a structure is illustrated for the strongest feature in the phosphorescence around $12150 \mathrm{~cm}^{-1}$ in Fig. 2a. At least eight lines spread over $90 \mathrm{~cm}^{-1}$ are recognized and the individual lines have widths of about $4 \mathrm{~cm}^{-1}$. All bands in the phosphorescence show the same multiplet structure, i.e. line splitting and intensity distribu- tion, except for the electronic origin. For the $0-0$ transition (Fig. 2b) a similar splitting is present but the intensity ratio of the various lines is different from that for the vibronic multiplets. For the fluorescence the observations are qualitatively alike. Multiplets spread over $120 \mathrm{~cm}^{-1}$ are seen for all bands with a characteristic intensity distribution for all vibronic bands (Fig. 2c) and a different one for the origin (Fig. 2d).

The observations represented in Figs. 1d and 2 are reminiscent of the Shpol'skii effect [15], previously reported for $C_{70}$ in a crystalline toluene matrix [14]. Upon cooling the n-pentane solution, rapid enough to avoid clustering of all $\mathrm{C}_{70}$ molecules, probably a glassy matrix is formed with crystalline regions. Within these microcrystals $\mathrm{C}_{70}$ molecules apparently occupy well-defined sites with a weak electron-phonon coupling and, correspondingly, the luminescence spectrum consists of narrow lines. The exceptional position of n-pentane compared to the longer n-alkanes may be related to the approximate match between the van der Waals dimensions of solvent and solute molecule. The length of n-pentane amounts to $7.4 \AA$ and the width of $C_{70}$ to $7.0 \AA$, while
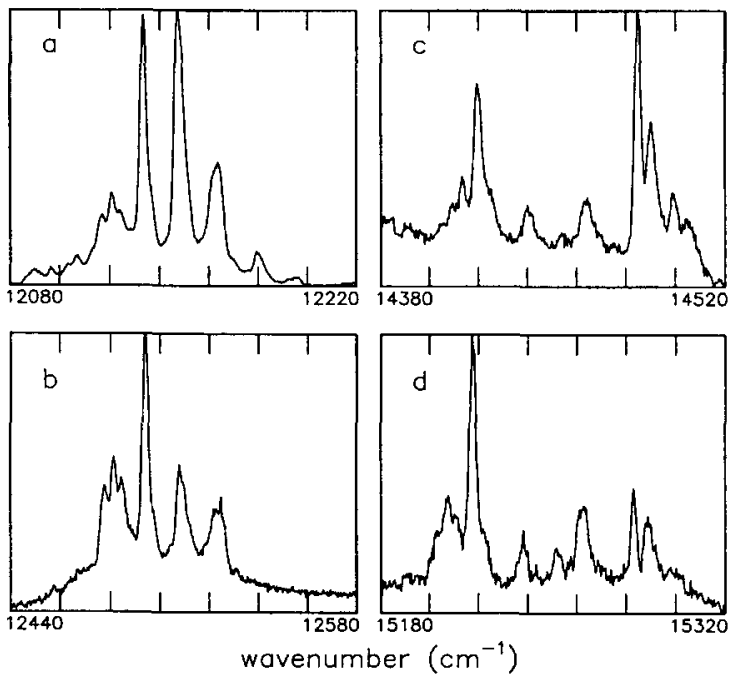

Fig. 2. Multiplets observed in the emission at $1.5 \mathrm{~K}$ of $\mathrm{C}_{70}$ in $\mathrm{n}$-pentane upon cooling quickly and exciting at $514 \mathrm{~nm}$. (a) A typical multiplet of a vibronic band in the phosphorescence, (b) the phosphorescence onset, (c) a typical multiplet of a vibronic band in the fluorescence, and (d) the fluorescence onset. 
comparison of the molecular volumes suggests that one $\mathrm{C}_{70}$ molecule might replace two n-pentane molecules.

The $\mathrm{C}_{70}$ molecules are incorporated in a limited number of ways. These distinct sites make up for the multiplet structure of each band (see Fig. 2) owing to a slight variation in the solute-solvent interaction from site to site. The multiplets in the fluorescence cover a wider frequency range than those in the phosphorescence and there is no straight one-to-one relation between the lines in the fluorescence and phosphorescence multiplets. As the intensity distribution within the multiplets is the same for all vibronic bands in the phosphorescence, we conclude that this distribution reflects the number of $\mathrm{C}_{70}$ molecules in the various sites. The same reasoning holds true for the fluorescence and the resulting multiplet structure is different from that for the phosphorescence because the coupling of singlet-excited $\mathrm{C}_{70}$ to the solvent matrix need not be the same as that of triplet-excited $C_{70}$. The distinct intensity patterns of vibronic and origin multiplets indicate that the mechanism by which the vibronic bands and the $0-0$ transition get their intensity is different. This probably means that we have to do with intrinsically symmetry $\left(D_{5 h}\right)$-forbidden transitions (vide infra). In that case the intensity of the $0-0$ transition depends on the (site-dependent) interaction with the environment of lower symmetry while the vibronic bands may acquire intensity through intramolecular (siteindependent) vibronic coupling.

When shifting the laser excitation towards the absorption onset, site selection has been observed with a concomitant simplification of the lumines-

Table 1

Band positions and intensities in the luminescence spectrum of a $5 \times 10^{-6} \mathrm{M}$ solution of $\mathrm{C}_{70}$ in n-pentane at $1.5 \mathrm{~K}$ upon excitation at 656 $\mathrm{nm}\left(15235 \mathrm{~cm}^{-1}\right)$. The positions $(\sigma)$ and relative positions with respect to the electronic origin $(\Delta \sigma)$ are listed in wavenumbers. The accuracy is about $1 \mathrm{~cm}^{-1}$, slightly worse for the band at $14921.1 \mathrm{~cm}^{-1}$. The intensity is given relative to that of the strongest vibronic band. The onset and the first vibronic band of the fluorescence were too close to the laser frequency and could not be seen during site selection; the positions of these bands have been inferred from the fluorescence spectrum upon excitation at $514 \mathrm{~nm}$. IR or R in columns 4 and 8 indicate that that particular frequency corresponds to an infrared-or Raman-active mode, respectively (from Refs. [17,21-26])

\begin{tabular}{|c|c|c|c|c|c|c|c|}
\hline \multicolumn{4}{|c|}{ Fluorescence } & \multicolumn{4}{|c|}{ Phosphorescence } \\
\hline \multirow{3}{*}{$\frac{\sigma\left(\mathrm{cm}^{-1}\right)}{15217.6}$} & $\Delta \sigma\left(\mathrm{cm}^{-1}\right)$ & \multicolumn{2}{|l|}{ Rel. int. (\%) } & \multirow{2}{*}{$\begin{array}{l}\sigma\left(\mathrm{cm}^{-1}\right) \\
12494.8\end{array}$} & \multirow[t]{2}{*}{$\Delta \sigma\left(\mathrm{cm}^{-1}\right)$} & \multicolumn{2}{|l|}{ Rel. int. (\%) } \\
\hline & & 36.0 & & & & 14.3 & \\
\hline & & & & 12221.2 & 273.6 & 2.0 & \\
\hline \multirow[t]{3}{*}{14921.1} & 296.5 & 9.5 & & & & & \\
\hline & & & & 12134.1 & 360.7 & 100.0 & $\mathbf{R}$ \\
\hline & & & & 12102.9 & 391.9 & 6.7 & \\
\hline 14797.5 & 420.1 & 28.0 & & 12076.0 & 418.8 & 3.8 & \\
\hline \multirow[t]{3}{*}{14757.0} & 460.6 & 4.0 & $\mathrm{IR} / \mathrm{R}$ & 12035.4 & 459.4 & 5.7 & IR $/ \mathrm{R}$ \\
\hline & & & & 11982.0 & 512.8 & 2.0 & \\
\hline & & & & 11957.8 & 537.0 & 14.3 & IR \\
\hline \multirow[t]{2}{*}{14636.7} & 580.9 & 100.0 & IR & 11915.3 & 579.5 & 18.1 & IR \\
\hline & & & & 11872.5 & 622.3 & 4.8 & \\
\hline 14540.4 & 677.2 & 24.0 & IR & 11818.8 & 676.0 & 8.6 & IR \\
\hline \multirow{2}{*}{14505.9} & 711.7 & 10.0 & & 11784.7 & 710.1 & 7.6 & \\
\hline & & & & 11748.0 & 746.8 & 10.0 & \\
\hline I 4420.0 & 797.6 & 56.0 & IR & 11697.2 & 797.6 & 7.4 & IR \\
\hline \multirow{2}{*}{14128.4} & 1089.2 & 32.0 & & 11405.4 & 1089.4 & 38.6 & \\
\hline & & & & 11317.5 & 1177.3 & 27.6 & \\
\hline 13965.0 & 1252.6 & 15.2 & IR & & & & \\
\hline 13894.8 & 1322.8 & 2.0 & & 11171.8 & 1323.0 & 12.9 & \\
\hline \multirow[t]{2}{*}{13789.1} & 1428.5 & 3.2 & IR & & & & \\
\hline & & & & 11062.1 & 1432.7 & 2.7 & IR \\
\hline \multirow[t]{3}{*}{13735.4} & 1482.2 & 4.0 & & & & & \\
\hline & & & & 11002.5 & 1492.3 & 2.5 & \\
\hline & & & & 10930.1 & 1564.7 & 10.5 & $\mathbf{R}$ \\
\hline
\end{tabular}



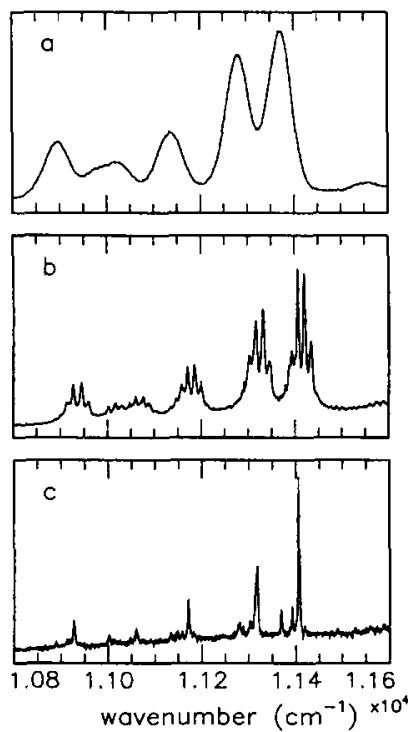

Fig. 3. Resolution enhancement and simplification of a part of the phosphorescence spectrum of $\mathrm{C}_{70}$. Spectrum of (a) a solution in $\mathrm{n}$-heptane upon excitation at $514 \mathrm{~nm}$ with linewidths of $90 \mathrm{~cm}^{-1}$, (b) a quickly cooled solution in n-pentane upon excitation of 514 $\mathrm{nm}$, and (c) a quickly cooled solution in n-pentane upon excitation at $656 \mathrm{~nm}$ with linewidths of $3 \mathrm{~cm}^{-1}$.

cence spectrum. Only one line of each multiplet in the fluorescence remains and by tuning the laser through the onset region we step from one line (i.e. site) to the other. A similar behaviour is observed for the phosphorescence but the selection appears less complete. For excitation at $656 \mathrm{~nm}\left(15235 \mathrm{~cm}^{-1}\right)$ all multiplets, both in fluorescence and in phosphorescence, reduce to a more or less single line. This is illustrated in Fig. 3 for some of the vibronic bands of the phosphorescence. Instead of a multiplet for each vibronic band upon excitation at $514 \mathrm{~nm}$ (Fig. 3b), one dominant feature with a width of $3 \mathrm{~cm}^{-1}$ is left for each vibronic band upon excitation at $656 \mathrm{~nm}$ accompanied by some smaller contributions from other sites (Fig. 3c). Comparison with the corresponding part of the phosphorescence spectrum in $\mathrm{n}$-heptane (Fig. 3a) reveals the gain in resolution achieved by site selection in the n-pentane Shpol'skii matrix. After elimination of the n-pentane Raman lines from the fluorescence region we are left with a luminescence spectrum from which the positions of the origin and the vibronic bands of fluorescence and phosphorescence have been obtained with a relative accuracy of about $1 \mathrm{~cm}^{-1}$. Frequencies of $12 \mathrm{vi-}$ bronic bands in the fluorescence and 19 in the phosphorescence with a relative intensity larger than $2 \%$ of the most intense band are summarised in Table 1. For the fluorescence the vibronic band 581 $\mathrm{cm}^{-1}$ from the $S_{1} \rightarrow S_{0}$ origin is most intense, for the phosphorescence one band stands out at 361 $\mathrm{cm}^{-1}$ from the $T_{1} \rightarrow S_{0}$ origin. The frequency shifts of the vibronic bands with respect to the electronic origins refer to vibrational quanta in the electronic ground state. The fluorescence and phosphorescence spectrum have at least eight vibrational frequencies below about $1500 \mathrm{~cm}^{-1}$ in common.

Convolution of the lines observed by site selection in n-pentane with a Gaussian envelope of 90 $\mathrm{cm}^{-1}$ width results in a vibronic intensity distribution similar to that observed in, e.g., n-heptane or methylcyclohexane. We infer from this that the description of the electronic structure of $\mathrm{C}_{70}$ in n-pentane to be given below may well apply for alkanes in general.

In order to find out about the electronic nature of $S_{1}$ and $T_{1}$, we consider which vibronic bands may occur in the luminescence spectrum for certain assignments. According to quantum-chemical calculations, the lowest excited singlet state $S_{1}$ of $C_{70}$ is expected to transform as either $A_{2}^{\prime}$ or $E_{1}^{\prime}[1,2]$. If $S_{1} \equiv E_{1}^{\prime}$, the $S_{1} \rightarrow S_{0}$ transition is electric-dipole allowed and $a_{1}^{\prime}$ vibrations may show up. This transi-

Table 2

Survey of symmetries of Herzberg-Teller active vibrational modes. The labels IR and $R$ indicate which modes are infrared- and Raman-active, respectively

\begin{tabular}{ll}
\hline Electronic transition & Symmetry of Herzberg-Teller active vibrational modes \\
\hline${ }^{1} A_{2}^{\prime} \rightarrow{ }^{3} A_{1}^{\prime}$ & $a_{1}^{\prime \prime}, e_{1}^{\prime}(I R)$ \\
${ }^{1} E_{1}^{\prime} \rightarrow{ }^{\prime} A_{1}^{\prime}$ & $a_{1}^{\prime}(R), a_{2}^{\prime}, e_{1}^{\prime \prime}(R), e_{2}^{\prime}(R)$ \\
${ }^{3} A_{2}^{\prime} \rightarrow{ }^{\prime} A_{1}^{\prime}$ & $a_{1}^{\prime \prime}, a_{2}^{\prime \prime}(I R), e_{1}^{\prime}(I R), e_{2}^{\prime \prime}$ \\
${ }^{3} E_{1}^{\prime} \rightarrow{ }^{\prime} A_{1}^{\prime}$ & $a_{1}^{\prime}(R), a_{2}^{\prime}, e_{1}^{\prime \prime}(R), e_{2}^{\prime}(R), e_{2}^{\prime \prime}$ \\
\hline
\end{tabular}


tion may well be weak and gain intensity through Herzberg-Teller coupling. The symmetry of the vibronic modes that could be involved is given in Table 2. If, on the other hand, $S_{1} \equiv A_{2}^{\prime}$, the $S_{1} \rightarrow S_{0}$ transition is electric-dipole forbidden and vibronic bands may acquire intensity through Herzberg-Teller coupling (see Table 2). In particular, intensity may be borrowed from allowed $E_{1}^{\prime} \rightarrow A_{1}^{\prime}$ transitions via coupling modes of $\mathrm{e}_{1}^{\prime}$ symmetry. The HerzbergTeller active modes for the transition from ${ }^{3} \mathrm{~A}_{2}^{\prime}$ or ${ }^{3} E_{1}^{\prime}$ to the totally symmetric ground state are listed in Table 2 as well. For a molecule of $D_{5 h}$ symmetry, IR-active modes belong to $\mathrm{a}_{2}^{\prime \prime}$ or $\mathrm{e}_{1}^{\prime}$ irreducible representations and Raman-active modes to $a_{1}^{\prime}, e_{1}^{\prime \prime}$ or $e_{2}^{\prime}$ irreducible representations. Consideration of Table 2 reveals that, as long as the Herzberg-Teller vibronic coupling scheme is appropriate, IR-active modes are expected in the luminescence only if the lowest excited state (singlet or triplet) has $\mathrm{A}_{2}^{\prime}$ orbital symmetry, and Raman-active modes only for $E_{1}^{\prime}$ orbital symmetry. Besides, Jahn-Teller active modes $\left(\mathrm{e}_{1}^{\prime}\right.$ or $\mathrm{e}_{2}^{\prime}$ ) may be present in the luminescence spectrum.

We can only present a tentative description of the vibronic structure of fluorescence and phosphorescence because knowledge of the vibrations of $\mathrm{C}_{70}$ is still limited. Various calculations of the 122 normal modes have led to different results [16-20] and a clear-cut assignment of the vibrational frequencies in infrared and Raman spectra to particular symmetry species is missing. Of the other vibrations even the frequencies are not known accurately enough to be of help in the interpretation of the luminescence spectra. In our description we only make use of experimental data $[17,21-26]$. On the basis of reported infrared and Raman spectra we have identified a number of vibrational quanta in fluorescence and phosphorescence as corresponding to infraredor Raman-active modes. This is indicated in Table 1. A band at $459 \pm 1 \mathrm{~cm}^{-1}$ shows up with considerable intensity both in infrared and Raman spectra. Other vibrational modes in the luminescence, in particular those of $420,513,747,1089,1177,1323$, and 1492 $\mathrm{cm}^{-1}$, have been observed as weak bands both in infrared and in Raman spectra. One should note that there is, as far as we know, no infrared data available below $400 \mathrm{~cm}^{-1}$.

As seen in Table 1, the most intense vibronic band of the fluorescence at $581 \mathrm{~cm}^{-1}$ from the origin concerns an IR-active mode and the same holds for at least five of the other vibronic bands. As outlined above, this suggests that the first excited singlet state is of $A_{2}^{\prime}$ character. The fluorescence origin is relatively strong in view of the $\mathrm{A}_{2}^{\prime}$ assignment of $S_{1}$. We presume that the electronic origin gets its intensity through matrix-induced static perturbations lowering the symmetry and mixing the close-lying $A_{2}^{\prime}$ and $E_{1}^{\prime}$ states. This would account for the strong dependence of the origin intensity on solvent as noticed earlier [13], and for the different intensity distribution among the multiplet lines of origin and vibronic bands. Preliminary emission experiments as a function of temperature reveal the occurrence of an additional fluorescence spectrum at higher temperatures in which the origin is the most prominent band. This is consistent with observations by Argentine and coworkers [12,13] and suggests to us that the $S_{2} \rightarrow S_{0}$ transition is electric-dipole allowed, which points to an $E_{1}^{\prime}$ character of $S_{2}$.

The many vibronic bands corresponding to IR-active modes in the phosphorescence (see Table 1) point to an $\mathrm{A}_{2}^{\prime}$ orbital character of the lowest triplet state as well (see Table 2). However, the most intense vibronic band at $361 \mathrm{~cm}^{-1}$ from the origin seems to concern a Raman-active mode and the same applies to the $1565 \mathrm{~cm}^{-1}$ band which indicates $E_{1}^{\prime}$ orbital character of $T_{1}$. This suggests a mixed character of the lowest triplet state and the present picture might well turn out to be too naive. A further description of the luminescence spectra and of the lowest electronic states of $\mathrm{C}_{70}$ requires a theoretical analysis of the expected vibronic intensity distribution in fluorescence and phosphorescence.

\section{Conclusion}

The luminescence spectrum of a low-concentrated solution of $\mathrm{C}_{70}$ in n-pentane after quickly cooling to liquid-helium temperatures shows a superior resolution. Site selection, observed for excitation at the absorption onset, results in linewidths of $3 \mathrm{~cm}^{-1}$ and largely simplifies both the fluorescence and the phosphorescence spectrum. The vibronic intensity distribution of the fluorescence points to an $\mathrm{A}_{2}^{\prime}$ character of the lowest excited singlet state. The presence of intensity at the origin reveals that $S_{1}$ has 
$E_{1}^{\prime}$ character as well, which most probably results from the mixing of $S_{1}\left(A_{2}^{\prime}\right)$ with the close-lying $S_{2}$ $\left(E_{1}^{\prime}\right)$ in an environment of symmetry lower than $D_{5 h}$.

\section{Acknowledgement}

This work forms part of the research program of the 'Stichting voor Fundamenteel Onderzoek der Materie' (FOM) and has been made possible by the financial support from the "Netherlands Organisation for Scientific Research' (NWO).

\section{Note added in proof}

While this paper was refereed we became aware of a paper by Negri and Orlandi (J. Phys. B., submitted) which describes the vibronic structure of the phosphorescence of $\mathrm{C}_{70}$ from a theoretical point of view. According to their calculations the most intense feature in our phosphorescence spectrum at $361 \mathrm{~cm}^{-1}$ from the origin probably concerns an infrared-active $\left(\mathrm{e}_{1}^{\prime}\right)$ mode as well. We thank professor Orlandi for sending us a presprint of their paper.

\section{References}

[1] G.E. Scuseria, Chem. Phys. Lett. 180 (1991) 451.

[2] J. Baker, P.W. Fowler, P. Lazzeretti, M. Malagoli and R. Zanasi, Chem. Phys. Lett. 184 (1991) 182.

[3] J. Feng, J. Li, Z. Li and M.C. Zerner, Intern. J. Quantum Chem. 39 (1991) 331.

[4] J. Fulara, M. Jakobi and J.P. Maier, Chem. Phys. Lett. 206 (1993) 203.

[5] S. Leach, in: Physics and chemistry of the fullerenes, ed. K. Prassides (Kluwer, Dordrecht, 1994) p. 117.

[6] D.J. van den Heuvel, G.J.B, van den Berg, E.J.J. Groenen, J.
Schmidt, I. Holleman and G. Meijer, J. Phys. Chem. 99 (1995) 11644.

[7] J.W. Arbogast and C.S. Foote, J. Am. Chem. Soc. 113 (1991) 8886.

[8] K. Palewska, J. Sworakowski, H. Chojnacki, E.C. Meister and U.P. Wild, J. Phys. Chem. 97 (1993) 12167.

[9] E. Shin, J. Park, M. Lee, D. Kim, Y.D. Suh, S.I. Yang, S.M. Jin and S.K. Kim, Chem. Phys. Lett. 209 (1993) 427.

[10] Y. Zeng, L. Biczok and H. Linschitz, J. Phys. Chem. 96 (1992) 5237.

[11] Y. Wang, J. Phys. Chem. 96 (1992) 764.

[12] S.M. Argentine, A.H. Francis, C.-C. Chen, C.M. Lieber and J.S. Siegel, J. Phys. Chem. 98 (1994) 7350.

[13] S.M. Argentine, K.T. Kotz and A.H. Francis, J. Am. Chem. Soc. 117 (1995) 11762.

[14] B.S. Razbirin, A.N. Starukhin, A.V. Chugreev, Yu.S. Grushko and S.N. Kolesnik, JETP Lett. 60 (1994) 451.

[15] E.V. Shpol'skii, Sov. Phys. Usp. 6 (1963) 411.

[16] P. Procacci, G. Cardini, P.R. Salvi and V. Schettino, Chem. Phys. Lett. 195 (1992) 347.

[17] R.A. Jishi, M.S. Dresselhaus, G. Dresselhaus, K. Wang, P. Zhou, A.M. Rao and P.C. Eklund, Chem. Phys. Lett. 206 (1993) 187; R.A. Jishi, R.M. Mirie, M.S. Dresselhaus, G. Dresselhaus and P.C. Eklund, Phys. Rev. B 48 (1993) 5634.

[18] G. Onida, W. Andreoni, J. Kohanoff and M. Parrinello, Chem. Phys. Lett. 219 (1994) 1.

[19] Y. Shinohara, R. Saito, T. Kimura, G. Dresselhaus and M.S. Dresselhaus, Chem. Phys. Lett. 227 (1994) 365.

[20] D. Inomata, N. Kurita, Sh. Suzuki and K. Nakao, Phys. Rev. B 51 (1995) 4533.

[21] D.S. Bethune, G. Meijer, W.C. Tang, H.J. Rosen, W.G. Golden, H. Seki, C.A. Brown and M.S. de Vries, Chem. Phys. Lett. 179 (1991) 181.

[22] J.P. Hare, J. Dennis, H.W. Kroto, R. Taylor, A.W. Allaf, S. Balm and D.R.M. Walton, J. Chem. Soc. Chem. Commun. (1991) 412.

[23] T.J. Dennis, J.P. Hare, H.W. Kroto, R. Taylor, D.R.M. Walton and P.J. Hendra, Spectrochim. Acta 47A (1991) 1289.

[24] P.H.M. van Loosdrecht, M.A. Verheijen, H. Meekes, P.J.M. van Bentum and G. Meijer, Phys. Rev. B 47 (1993) 7610.

[25] P. Bowmar, W. Hayes, M. Kurmoo, P.A. Pattenden, M.A. Green, P. Day and K. Kikuchi, J. Phys. Condens. Matter 6 (1994) 3161.

[26] A. von Czarnowski and K.H. Meiwes-Broer, Chem. Phys. Lett. 246 (1995) 321. 